\title{
A challenge for the procedural deficit hypothesis: how should we measure sequential learning in childhood?
}

\author{
Saloni Krishnan and Kate E. Watkins \\ Department of Experimental Psychology, University of Oxford
}

\author{
Note $-X X X$ to be replaced by citation to the authors of the commentary
}

A recent publication by West and colleagues (2018) highlighted the fact that many tasks used to measure implicit learning in developmental populations are unreliable. As indicated by the title of their paper, West and colleagues suggest this is a problem for the procedural deficit hypothesis (a theory that suggests that procedural learning underlies the impairment in those with developmental language disorders (DLD), Ullman \& Pierpont, 2005). In a recent commentary, XXX argue that the experiment conducted by West et al. was an inadequate test of the procedural deficit hypothesis. Here, we evaluate both points of view and conclude that we need better measurements of sequential learning in childhood.

XXX point out that West et al. (2018) used a sample that mainly comprises the typically developing population and indeed this is the strongest point of their critique. To fully criticise the procedural deficit hypothesis $(\mathrm{PDH})$, we need to test children with language disorders, or at least at the lower end of the language learning spectrum. Indeed, it is possible that only those with low language learning ability exhibit particular problems with procedural learning. Not having sampled from children with poor performance on language tests could have reduced the variation seen in implicit learning tasks, and therefore limit correlations with language abilities.

To test their construct of implicit/procedural memory, West et al. used a serial reaction time (SRT) task, a contextual cueing task, and a Hebb repetition task. This is because West et al. use the terms implicit and procedural interchangeably, focusing only on awareness of the material to be learned. The origins of the dissociation between declarative and procedural memory, or explicit and implicit memory, come from neuropsychological studies of adult patients with damage to the medial temporal lobe (see Squire \& Dede, 2015 for a review of these ideas). These patients were found to be impaired on declarative tasks involving scenes, faces, and words where knowledge was explicit, but not on implicit tasks where learning was expressed through performance, such as skill learning (for example, mirror drawing or serial reaction time tasks), perceptual priming, or conditioning. These implicit tasks are argued to depend on fronto-striatal, neocortical, and cerebellar systems, respectively (Squire \& Dede, 2015). As Ullman \& Pierpont (2005) specifically define procedural memory tasks as those that primarily rely on a network rooted in the basal ganglia (i.e. part of the frontostriatal system), only the SRT, and not contextual cueing or Hebb repetition, would be expected to tap into the function of this system. Even so, it needs to be acknowledged that the dichotomy between declarative and procedural systems is not quite so absolute. Even tasks like the SRT have declarative components (Robertson, 2007; Schendan, Searl, Melrose, \& Stern, 2003). Taking all this into consideration, we need clearer definitions of what kind of learning and of what kind of material is being measured by a behavioural task. One possibility is that the perceptual detection of regularities is the fundamental learning impairment. Both SRT tasks and statistical learning tasks (as used in Evans, Saffran, \& Robe-Torres, 2009) would test this. 
Alternatively, the performance element that accompanies some (but not all) of implicit learning tasks could be at the core of the impairment.

The limitations outlined above do not negate the point that West and colleagues make about the reliability of tasks they used to measure implicit learning, including verbal and non-verbal versions of the serial reaction time task. In their study, reliability estimates ranged from .00 to .24 . One reason these reliabilities are so low is because the implicit learning tasks they used depend on changes in reaction times, which are noisy measures. The lack of reliability is likely to be exacerbated in developmental populations when it is necessary to have short tasks with relatively few trials because of limitations in concentration and remaining "on task". To address some of these issues, Kuppuraj et al (2018) recently developed an online statistical learning task (without the performance element of the SRT) and achieved a test-retest reliability of .67 in adults. This suggests that it is possible to tackle some of issues related to reliability in future implicit learning research, but it is necessary to test this in children specifically.

The PDH has been a catalyst for the field of developmental language disorders to focus on learning profiles in this population. As XXX note, a number of studies from different research groups have noted differences between individuals with DLD and those without on tasks that involve learning regularities in sequences (Lum, ContiRamsden, Morgan, \& Ullman, 2014). However, the lack of reliability of implicit learning measures is of concern, especially given the known issue of publication bias for positive findings. Other studies with similar sample sizes indicate performance on other implicit learning tasks such as pursuit rotor ability or conditioning is relatively good in DLD (Hardiman, Hsu, \& Bishop, 2013; Hsu \& Bishop, 2014). Furthermore, there is considerable evidence indicating that the learning of phonological sequences is impaired in those with DLD (Bishop \& Hsu, 2015; Coady \& Evans, 2008). Consequently, there remain theoretical reasons to investigate why the learning of sequences is an area of specific impairment in DLD, and why this impairment is substantially affects the articulatory motor system and not the hands or limbs. This is especially relevant when taken alongside findings from brain imaging studies that fronto-striatal circuitry is affected in individuals with DLD, although we note that this is also an area characterised by small samples and inconsistency in findings (Krishnan, Watkins, \& Bishop, 2016; Mayes, Reilly, \& Morgan, 2015). If we find there is a core implicit sequential learning deficit in DLD, it will influence the delivery of language training, particularly whether we explicitly teach words and grammatical structures. This leaves an important challenge for the field: how can we capture individual differences in sequence learning in a reliable manner? What adaptations should we make for a developmental population, and especially for those that might struggle to understand complex instructions? This is crucial to addressing how these abilities are related to language learning. In the future, the use of open science practices such as pre-registering specific hypotheses and methods, in combination with development of robust and reliable tasks, may allow us to address these questions.

\section{References}

Bishop, D. V. M., \& Hsu, H. J. (2015). The declarative system in children with specific

language impairment: a comparison of meaningful and meaningless auditory-visual 
paired associate learning. BMC Psychology, 3(1), 3. https://doi.org/10.1186/s40359015-0062-7

Coady, J. A., \& Evans, J. L. (2008). Uses and interpretations of non-word repetition tasks in children with and without specific language impairments (SLI). International Journal of Language \& Communication Disorders, 43(1), 1-40. https://doi.org/10.1080/13682820601116485

Evans, J. L., Saffran, J. R., \& Robe-Torres, K. (2009). Statistical Learning in Children With Specific Language Impairment. Journal of Speech, Language, and Hearing Research, 52(2), 321-335. https://doi.org/10.1044/1092-4388(2009/07-0189)

Hardiman, M. J., Hsu, H., \& Bishop, D. V. M. (2013). Children with Specific Language Impairment are not impaired in the acquisition and retention of Pavlovian delay and trace conditioning of the eyeblink response. Brain and Language, 127(3), 428-439. https://doi.org/10.1016/j.bandl.2013.08.001

Hsu, H. J., \& Bishop, D. V. M. (2014). Sequence-specific procedural learning deficits in children with specific language impairment. Developmental Science, 17(3), 352-365. https://doi.org/10.1111/desc.12125

Krishnan, S., Watkins, K. E., \& Bishop, D. V. M. (2016). Neurobiological Basis of Language Learning Difficulties. Trends in Cognitive Sciences, 20(9), 701-714. https://doi.org/10.1016/j.tics.2016.06.012

Kuppuraj, S., Duta, M., Thompson, P., \& Bishop, D. (2018). Online incidental statistical learning of audiovisual word sequences in adults: a registered report. Royal Society Open Science, 5(2), 171678. https://doi.org/10.1098/rsos.171678 
Lum, J. A. G., Conti-Ramsden, G., Morgan, A. T., \& Ullman, M. T. (2014). Procedural learning deficits in specific language impairment (SLI): A meta-analysis of serial reaction time task performance. Cortex, 51, 1-10. https://doi.org/10.1016/j.cortex.2013.10.011

Mayes, A. K., Reilly, S., \& Morgan, A. T. (2015). Neural correlates of childhood language disorder: a systematic review. Developmental Medicine \& Child Neurology, 57(8), 706-717. https://doi.org/10.1111/dmcn.12714

Robertson, E. M. (2007). The Serial Reaction Time Task: Implicit Motor Skill Learning? Journal of Neuroscience, 27(38), 10073-10075. https://doi.org/10.1523/JNEUROSCI.2747-07.2007

Schendan, H. E., Searl, M. M., Melrose, R. J., \& Stern, C. E. (2003). An fMRI Study of the Role of the Medial Temporal Lobe in Implicit and Explicit Sequence Learning. Neuron, 37(6), 1013-1025. https://doi.org/10.1016/S0896-6273(03)00123-5

Squire, L. R., \& Dede, A. J. O. (2015). Conscious and Unconscious Memory Systems. Cold Spring Harbor Perspectives in Biology, 7(3), a021667. https://doi.org/10.1101/cshperspect.a021667

Ullman, M. T., \& Pierpont, E. I. (2005). Specific Language Impairment is not Specific to Language: the Procedural Deficit Hypothesis. Cortex, 41(3), 399-433. https://doi.org/10.1016/S0010-9452(08)70276-4

West, G., Vadillo, M. A., Shanks, D. R., \& Hulme, C. (2018). The procedural learning deficit hypothesis of language learning disorders: we see some problems. Developmental Science, 21(2), e12552. https://doi.org/10.1111/desc.12552 